\title{
Semiconductor quantum tubes: Dielectric modulation and excitonic response
}

\author{
David Kammerlander, ${ }^{1,2, *}$ Filippo Troiani, ${ }^{1}$ and Guido Goldoni ${ }^{1,2}$ \\ ${ }^{1}$ Centro S3, CNR-Istituto di Nanosciencze, Via Campi 213A, I-41100 Modena, Italy \\ ${ }^{2}$ Dipartimento di Fisica, Università di Modena e Reggio Emilia, Via Campi 213/A, 41100 Modena, Italy
}

(Received 26 October 2009; revised manuscript received 19 January 2010; published 9 March 2010)

\begin{abstract}
We study theoretically the optical properties of quantum tubes, one-dimensional semiconductor nanostructures where electrons and holes are confined to a cylindrical shell. In these structures, which bridge between two-dimensional and one-dimensional systems, the electron-hole interaction may be modulated by a dielectric substance outside the quantum tube and possibly inside its core. We use the exact Green's function for the appropriate dielectric configuration and exact diagonalization of the electron-hole interaction within an effective-mass description to predict the evolution of the exciton binding energy and oscillator strength. Contrary to the homogeneous case, in dielectrically modulated tubes, the exciton binding is a function of the tube diameter and can be tuned to a large extent by structure design and proper choice of the dielectric media.

DOI: 10.1103/PhysRevB.81.115310

PACS number(s): 73.22.Lp, 78.67.Ch
\end{abstract}

\section{INTRODUCTION}

Cylindrical semiconductor nanostructures bridge between quasi-one-dimensional (1D) systems at small diameters and quasi-two-dimensional (2D) systems in the opposite limit, thus extending the wealth of physics and applications of lowdimensional solid-state systems. The controlled growth of semiconductor quantum tubes (QTs) with diameters in the $10-100 \mathrm{~nm}$ range has been recently demonstrated through several techniques, including multilayer overgrowth of nanowires $^{1-3}$ and strain-induced bending of a planar heterostructure. ${ }^{4,5}$ In addition to QTs with a solid semiconductor core, it is possible to grow hollow QTs, where the charge carriers are confined in a thin semiconductor shell, encompassed by a barrier material which is only a few $\mathrm{nm}$ thick. ${ }^{6-8}$ Large surface-to-volume ratios and the possibility of various functionalizations on both the internal and external surfaces make the latter systems particularly interesting for applications. ${ }^{9}$

Although experiments concerning the optical properties of these systems are still limited, advancements in the optical quality of the samples point to a rapid increase of these investigations..$^{2,10-12}$ The excitonic properties of semiconductor QTs are particularly interesting with respect to conventional semiconductor quantum wires, where excitons are confined in the core of the nanostructure. ${ }^{13-20}$ On the one hand, due to the combined effect of the QT curvature and of the quasi-2D confinement of carriers in the cylindrical shell, excitonic binding energies might be substantially stronger than in bulk even for large diameter QTs. On the other hand, a dielectric medium outside the shell of the QT may result in a dielectric confinement of the electric field felt by the optically excited electron-hole pairs, in most cases enhancing their excitonic binding energy. Since the dielectric interface is spatially separated from the carriers, which are confined deep inside the shell, excitonic binding and sensitivity to the medium might be strongly enhanced without spoiling the optical properties of the electronic system, ${ }^{21}$ analogously to core-shell nanowires. ${ }^{12}$ The screening provided by the dielectric environment can be varied in a broad range. ${ }^{22}$ The tunability of the dielectric constant in the core of the QT, obtained, e.g., by oxidation, ${ }^{23}$ can further increase such effects.

Present work on QTs theoretically considered magnetic states, ${ }^{24,25}$ reported experimental evidence of the AharonovBohm effect, ${ }^{26}$ and treated optical properties, ${ }^{27}$ but the influence of the dielectric mismatch between the nanostructure and the environment has been studied so far only for conventional quantum wires $^{21}$ and freestanding nanowires. ${ }^{20}$ Here we will consider also a dielectric mismatch between the core and the shell, which will lead to a considerable change in the electron-hole interaction as shown in Fig. 2.

Hereafter, we investigate the excitonic binding and oscillator strength in hollow and filled QTs for different geometries and dielectric configurations. Besides increasing due to the reduced screening, the excitonic binding strongly depends on the QT diameter and on the dielectric medium. The paper is organized as follows. In Sec. II, we outline the theoretical model, which includes the exact solution of the Poisson equation and the diagonalization of the electron-hole Hamiltonian within the envelope-function approximation. In Secs. III and IV, we report our results and draw the conclusions, respectively.

\section{MODEL}

The system we consider consists of an infinite tube with cylindrical symmetry $^{28}$ (see Fig. 1). For simplicity, we assume that the motion in the radial direction is frozen and that charge carriers are radially confined in a $\delta$-like well at a distance $R$ from the tube axis. This electronic layer is buried in the middle of a coaxial cylindrical shell of thickness $2 \delta$ with dielectric constant $\epsilon_{S}$, while the core and the environment have in general different dielectric constants, $\epsilon_{C}$ and $\epsilon_{E}$, respectively. Since the shell is a semiconductor material, typically $\epsilon_{S} \geq \epsilon_{C}, \epsilon_{E}{ }^{22}$

The invariance under translations along and rotations around the tube axis warrant the separation of the center of mass and relative coordinates. The motion of the Wannier exciton $^{29}$ in the relative degrees of freedom is determined by the envelope-function Hamiltonian 


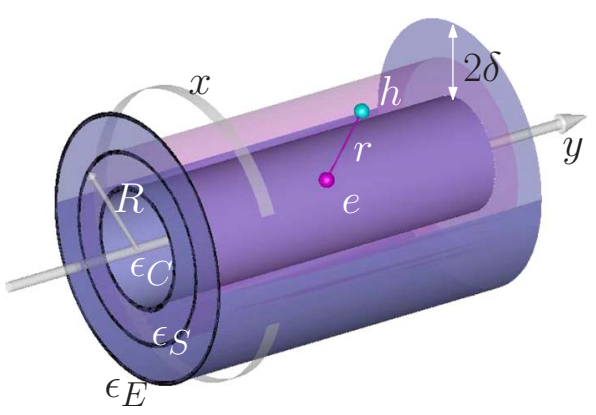

FIG. 1. (Color online) Schematics of a QT with outer surface cut open. The electron-hole pair moves on a cylindrical surface of radius $R$, embedded in a shell of thickness $2 \delta . x$ and $y$ are the relative electron-hole coordinates. The dielectric constants $\epsilon_{E}, \epsilon_{S}, \epsilon_{C}$ characterize the environment, the shell, and the core region, respectively.

$$
H(x, y)=-\frac{1}{2}\left[\frac{\partial^{2}}{\partial x^{2}}+\frac{\partial^{2}}{\partial y^{2}}\right]-V(x, y),
$$

expressed in units of the effective Hartree $\mathrm{Ha}^{*}=\left(\mu / \epsilon_{S}^{2}\right) \mathrm{Ha}$, with $\mu=m_{e} m_{h} /\left(m_{e}+m_{h}\right)$ the reduced electron-hole mass. The relative coordinates around the circumference $(x=R \phi)$ and along the tube axis ( $y$, see Fig. 1) are in units of the effective Bohr radius, $a_{B}^{*}=\left(\epsilon_{S} / \mu\right) a_{B}$.

The effective Coulomb interaction potential $V(x, y)$ between the confined electron and hole depends parametrically on the dielectric constants $\left(\epsilon_{C}, \epsilon_{S}, \epsilon_{E}\right)$ and on the tube geometry through $\delta$ and $R$. In cylindrical coordinates, the potential (scaled with $\left.\mathrm{Ha}^{*}\right)$ generated by a charge at $r^{\prime}=\left(\rho^{\prime}, \phi^{\prime}, z^{\prime}\right)$ reads

$$
\begin{aligned}
& V_{\alpha}\left(\boldsymbol{r}, \boldsymbol{r}^{\prime}\right)=\frac{\epsilon_{S}}{2 \pi^{2}} \sum_{m=-\infty}^{\infty} e^{l m\left(\phi-\phi^{\prime}\right)} \\
& \times \int_{0}^{\infty} d k \cos \left[k\left(z-z^{\prime}\right)\right] g_{m, \alpha}\left(k, \rho, \rho^{\prime}\right),
\end{aligned}
$$

where $\alpha=C, S, E$ indicates whether the position of the test charge $r=(\rho, \phi, z)$ is in the core, shell, or environment region, respectively, and $g_{m, \alpha}\left(k, \rho, \rho^{\prime}\right)$ is the solution of the radial Poisson equation in that region (see the Appendix for further details). The interaction $V$ in Eq. (1) coincides with $V_{S}$, with $\rho=\rho^{\prime}=R$. As shown in the Appendix,

$$
g_{m, S}(k, R, R)=\frac{4 \pi}{\epsilon_{S}}\left[\widetilde{B}_{m}^{<}+\widetilde{C}_{m}^{<}\right]\left[\tilde{B}_{m}^{>}+C_{m}^{>}\right] I_{m}(k R) K_{m}(k R),
$$

where $I_{m}, K_{m}$ are the Bessel functions of the first and second kinds; the coefficients $\widetilde{B}_{m}^{<}, \widetilde{C}_{m}^{<}, \widetilde{B}_{m}^{>}, C_{m}^{>}$are given in Eqs. (A10) and (A12) in terms of $I_{m}, K_{m}$ and their derivatives.

To illustrate how the electron-hole interaction is influenced by the dielectric environment, we show in Fig. 2 the potential $V$ for (i) a filled QT, with a core of the same material as the shell, immersed in a substance with a lowdielectric constant $\left(\epsilon_{C}=\epsilon_{S}=10 \epsilon_{E}\right)$, and (ii) a hollow QT, with the same low-dielectric constant substance inside and outside the shell $\left(\epsilon_{S}=10 \epsilon_{E}=10 \epsilon_{C}\right)$. For comparison, we also show

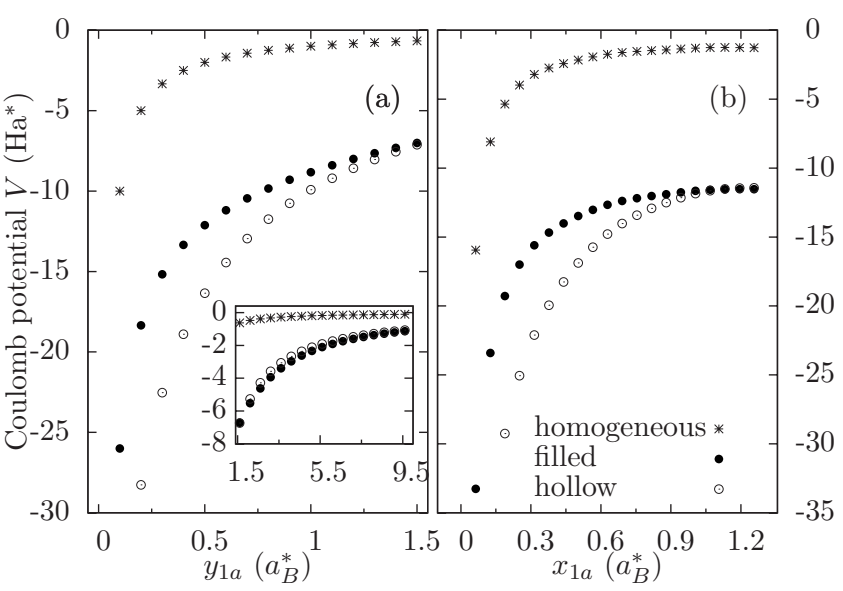

FIG. 2. Electrostatic interaction $V(x, y)$ between an electronhole pair confined to a cylindrical surface with diameter $D=0.8 a_{B}^{*}$, buried in a shell with thickness $2 \delta=0.1 a_{B}^{*}$ with dielectric mismatch, as follows. Homogeneous case: $\epsilon_{C}=\epsilon_{S}=\epsilon_{E}$. Filled case: $\epsilon_{C}=\epsilon_{S}$ $=10 \epsilon_{E}$. Hollow case: $\epsilon_{S}=10 \epsilon_{C}=10 \epsilon_{E}$. (a) Interaction along the QT, $V(x=0, y)$. (b) Interaction around the QT, $V(x, y=0)$. Inset in (a): $V(x=0, y)$ in a larger range of $y$.

the dielectrically homogeneous case $\left(\epsilon_{C}=\epsilon_{S}=\epsilon_{E}\right)$, where the $V$ reduces to the usual Coulomb potential $V(x, y)=$ $-1 / \epsilon_{S} \sqrt{[2 R \sin (x / 2 R)]^{2}+y^{2}}$. Figure 2(a) shows the interaction along the QT $V(x=0, y)$, while Fig. 2(b) shows the interaction around the cylinder $V(x, y=0)$. The Coulomb interaction for the hollow and filled cases is for all distances stronger than in the homogeneous case, since the average dielectric constant of the system is smaller and the electric field is not screened outside and, for the hollow case, also inside the QT. The interaction in the filled and hollow cases is substantially different only for distances smaller or comparable to the Bohr radius, with the interaction in the hollow case being stronger. For larger distances [inset of Fig. 2(a)], on the other hand, the nontrivial influence of the dielectric mismatch between the core and the shell leads to crossing of the potentials for hollow and filled QTs before both converge to the same value.

A convenient basis set to represent the exciton wave function is obtained by multiplying eigenfunctions of the linear momentum operator along $y\left(e^{i k y}\right)$ and of the angularmomentum operator along the tube axis $\left(e^{i n x / R}\right)$. Imposing periodic Born-von Karman boundary conditions along $y$, with period $L$ sufficiently larger than the effective Bohr radius of the material, results in $k=p \Delta k$, with $\Delta k=2 \pi / L$. The wave function thus reads

$$
\psi_{j}(x, y)=\frac{1}{2 \pi} \sqrt{\frac{\Delta k}{4 R}} \sum_{n=-N}^{N} \sum_{p=-P}^{P} C_{n, p}^{j} e^{i n x / R} e^{l p \Delta k y},
$$

where $p, n \in \mathbb{Z}$ and $j$ indicates the $j$ th exciton state. The coefficients $C_{n, p}^{j}$ are obtained from the Schrödinger equation in the above basis 
TABLE I. Parameters of materials under consideration. The mass, energy, and length are in units of bare electron mass, eV, and nm, respectively. Values are taken from Refs. 31, 40, and 41.

\begin{tabular}{lcccccc}
\hline \hline Material & $m_{e}$ & $m_{h}$ & $\epsilon_{S}$ & $\mathrm{Ha}^{*}$ & $a_{B}^{*}$ & $E_{g}$ \\
\hline InAs & 0.026 & 0.33 & 14.6 & 0.0031 & 32.05 & 0.35 \\
GaAs & 0.067 & 0.35 & 12.5 & 0.0098 & 11.76 & 1.43 \\
InP & 0.08 & 0.33 & 12.4 & 0.0123 & 9.23 & 1.34 \\
\hline \hline
\end{tabular}

$\sum_{n^{\prime}, p^{\prime}}\left\{\frac{1}{2}\left[\left(\frac{n^{\prime}}{R}\right)^{2}+\left(p^{\prime} \Delta k\right)^{2}\right] \delta_{n, n^{\prime}} \delta_{p, p^{\prime}}-U_{n^{\prime}, p^{\prime}}^{n, p}\right\} C_{n^{\prime}, p^{\prime}}^{j}=E_{j} C_{n, p}^{j}$.

The diagonal term in the first line represents the kinetic energy, whereas the matrix elements of the electron-hole Coulomb interaction term are given by

$$
U_{n^{\prime}, p^{\prime}}^{n, p}=\frac{\Delta k}{(2 \pi)^{2}} g_{\left|n-n^{\prime}\right|, S}\left(\left|p-p^{\prime}\right| \Delta k, R, R\right)
$$

In order to reduce the dimension of the Hamiltonian matrix, we introduce a cutoff energy $E_{\text {cut }}$, set the maximum number of plane waves $P=\sqrt{2 E_{\text {cut }}} / \Delta k$, and choose the maximum number of orbital modes $N$ in Eq. (4) as the nearest integer to $n(p)=R \sqrt{2 E_{\text {cut }}-(p \Delta k)^{2}}$. The Hamiltonian matrix is block diagonalized using a symmetrized basis set. In particular, we consider linear combinations of the above basis functions that are even or odd with respect to the inversion of the relative coordinates $x$ and $y$, which is the equivalent of inverting the absolute coordinates, since the corresponding inversion operators $\Pi_{x}, \Pi_{y}$ commute with the relative motion Hamiltonian

$$
\left[H, \Pi_{x}\right]=0, \quad\left[H, \Pi_{y}\right]=0 .
$$

The resulting energy $E_{j}$ is obtained with respect to the energy minimum of the conduction band. Therefore, the binding energy of the exciton ground state is $E_{b}^{x}=-E_{0}$. In the presence of a photon gauge field, the electron-hole pair recombines emitting a photon of energy $E_{g}-E_{b}^{x}$. The recombination rate is related to the dimensionless oscillator strength $f$, which in the dipole approximation reads ${ }^{30}$

$$
f=S_{0} \frac{\left|\psi_{0}(0,0)\right|^{2}}{E_{g}-E_{b}^{x}} \delta_{Q, 0} .
$$

Here, $\left|\psi_{0}(0,0)\right|^{2}$ is the envelope function of the exciton ground state given by Eq. (4), $\boldsymbol{Q}$ is the momentum of the center of mass, $E_{g}$ is the energy gap between valence and conduction bands, and $S_{0}=E_{p} / \epsilon_{S}$, where $E_{p}$ is the energy associated with Kane's matrix elements. ${ }^{31}$

\section{RESULTS}

In the following, we investigate the excitonic properties of QTs made of the direct-gap materials, InAs, GaAs, and InP, and two different dielectric configurations: filled QTs, with a core of the same material of the shell $\left(\epsilon_{C}=\epsilon_{S} \neq \epsilon_{E}\right)$, and hollow QTs, with the core of the same material as the environment $\left(\epsilon_{C}=\epsilon_{E} \neq \epsilon_{S}\right)$. We consider QTs with diameters in the 20-100 nm range and a constant shell thickness $2 \delta$ $=10 \mathrm{~nm}$, comparable to state-of-the-art samples., 1,32 Material parameters used in the calculations are listed in Table I.

In Fig. 3, we plot the energy of the exciton for hollow and filled QTs in vacuum $\left(\epsilon_{E}=1\right)$ and compare it to the 2D limit $R \rightarrow \infty$; this, in the case of excitons confined to a strictly 2D layer, is 4 times the bulk value. ${ }^{33-35}$ The exciton binding energy shows a strong increase with respect to the 2D limit and a marked diameter dependence, which is different for hollow and filled QTs: while in the former case the exciton binding energy becomes weakly dependent of the diameter for QTs larger than $\sim 60 \mathrm{~nm}$, the latter one shows a strong dependence even for the larger QTs.

It is instructive to contrast these results with the exciton energies of QTs which are dielectrically homogeneous, that is, buried in a material with the same bulk dielectric constant

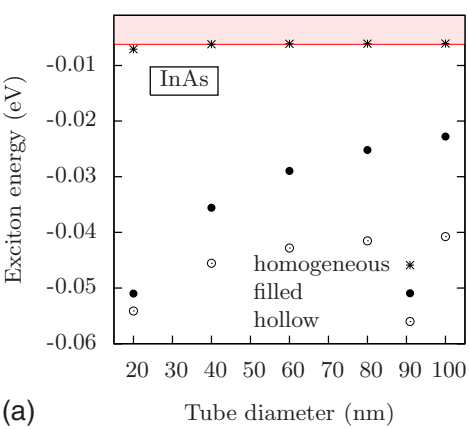

(a)

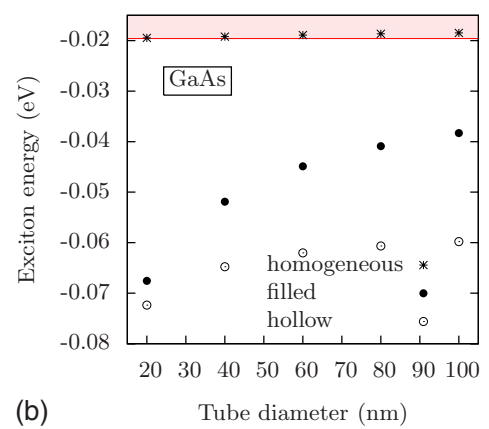

(b)

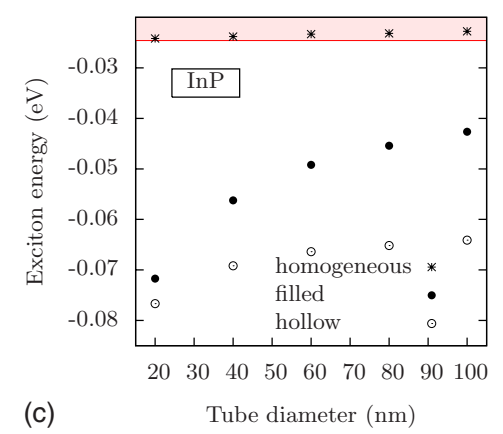

FIG. 3. (Color online) Ground state energy of the exciton for InAs, GaAs, and InP tubes. Different symbols correspond to a homogeneous dielectric constant $\left(\epsilon_{C}=\epsilon_{S}=\epsilon_{E}\right.$, crosses), a filled tube $\left(\epsilon_{C}=\epsilon_{S}, \epsilon_{E}=1\right.$, filled circles), and a hollow tube $\left(\epsilon_{C}=\epsilon_{E}=1\right.$, empty circles). Shaded region marks the analytical $2 \mathrm{D}$ limit for the homogenous case. The exciton energy for hollow and filled QTs increases as $\sim-10 / D$ and $\sim-10 / D^{2}$, respectively. 

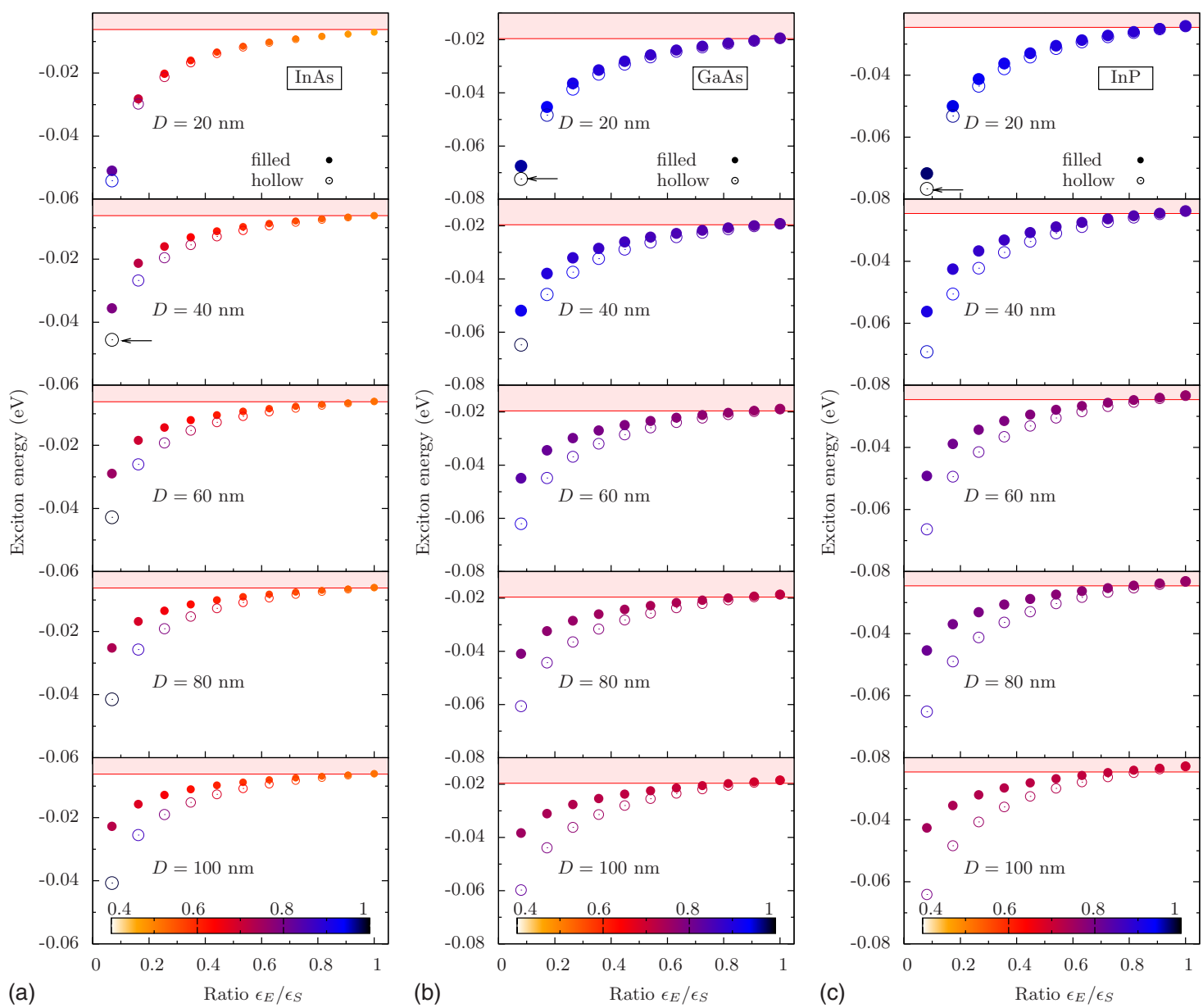

FIG. 4. (Color online) Ground state energy of the exciton for different values of the diameter $D$ as a function of the dielectric contrast $\epsilon_{E} / \epsilon_{S}$, with $\epsilon_{C}=\epsilon_{E}\left(\epsilon_{C}=\epsilon_{S}\right)$ for hollow (filled) QTs. Shaded area indicates the limit of excitons in a 2D quantum well. Color and size of the symbols (filled and empty circles for the respective QTs) are proportional to the relative oscillator strength, normalized to the maximum value for each material (marked with an arrow).

of the semiconductor shell $\left(\epsilon_{S}=\epsilon_{C}=\epsilon_{E}\right)$. In this case, exciton energies do not show any dependence on the diameter and are pinned to the $2 \mathrm{D}$ value (see Fig. 3). This is due to the small value of the Bohr radius with respect to the tube diameters shown here, so that the curvature of the surface plays only a minor role. ${ }^{36}$ Clearly, for smaller diameters (not shown here), the exciton binding energy increases and the exciton energy redshifts, since the binding energy is infinite in the strictly $1 \mathrm{D}$ limit. ${ }^{37,38}$ In Fig. 3, this can only be recognized in the tiny redshift for InAs at the smallest diameter.

The large binding energy of dielectrically modulated QTs with respect to homogeneous ones is an obvious consequence of the smaller screening of the electron-hole interaction in regions where the dielectric constant is 1. Accordingly, exciton energies of filled and hollow QTs are similar for the smaller diameters because their dielectric configuration differs only in the core, which is a small fraction of the volume if $2 \delta=10 \mathrm{~nm}$. Increasing the diameter while leaving the shell thickness constant corresponds to increasing the core region with respect to the shell, thus enhancing the difference between filled and hollow QTs. In both cases, energies are increasing with diameter due to the larger area occupied by the cross section of semiconductor shell, but faster for filled than for hollow QTs. This is consistent with the following argument: the cross section of the tube with $\epsilon_{S}$ is a ring of area $2 D \delta \pi$ for hollow QTs and a circle of area $(D / 2+\delta)^{2} \pi$ for filled ones. Thus, the screening area is growing faster for filled QTs. In fact, the exciton energy for hollow and filled QTs increases as $\sim-10 / D$ and $\sim-10 / D^{2}$, respectively.

All in all, excitons in QTs can be from twice (filled GaAs QTs, $D=100 \mathrm{~nm}$ ) up to 7 times (hollow InAs QTs at $D$ $=20 \mathrm{~nm}$ ) more strongly bound with respect to the respective 2D bulk exciton. Moreover, in all investigated cases, they have a binding energy which is much larger than thermal energy at room temperature.

In Fig. 3, we used the permittivity of vacuum, which leads to the largest possible dielectric confinement effects in a given configuration. Next, we show how the binding energy depends on the dielectric constant of the medium by which the QTs are surrounded. ${ }^{22,32,39}$ Figure 4 shows the binding energy as a function of the ratio $\epsilon_{E} / \epsilon_{S}$. The leftmost point on the horizontal axis corresponds to the case $\epsilon_{E}=1$, while the ratio $\epsilon_{E} / \epsilon_{S}=1$ corresponds to the homogeneous case, with the same dielectric constant $\epsilon_{S}$ filling all spaces. The general behavior is the same for the three materials. The difference in 

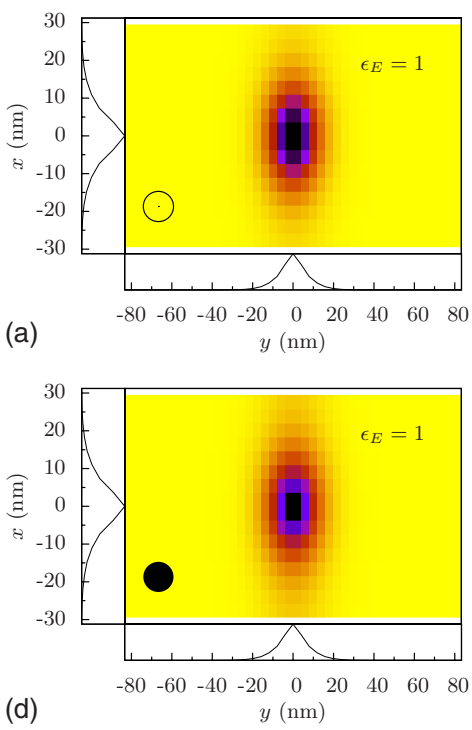
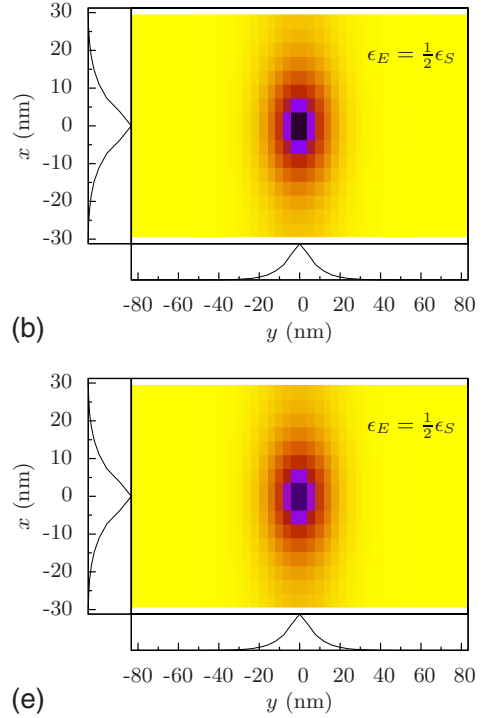
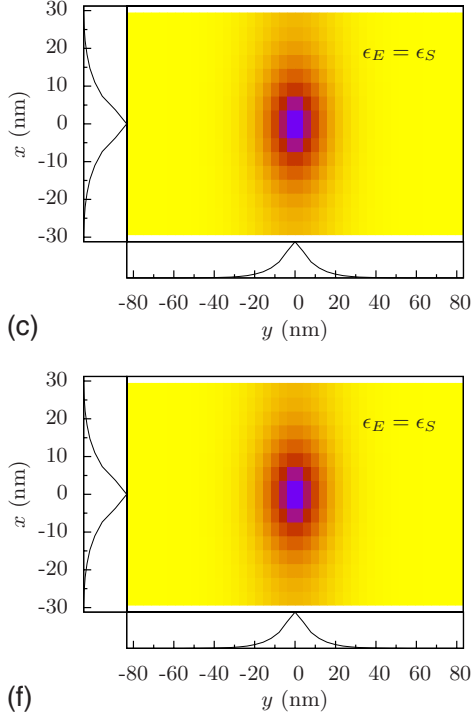

FIG. 5. (Color online) Wave function of the exciton ground state in InAs for tubes of diameter $D=20 \mathrm{~nm}$ and three different values of $\epsilon_{E}$. Top row (empty circle): hollow QTs, $\epsilon_{C}=\epsilon_{E}$. Bottom row (filled circle): filled QTs, $\epsilon_{C}=\epsilon_{S}$. For each panel, an inset on the left (bottom) shows the cut of the total wave function at $y=0(x=0)$. The $x$ axis extends from $-R \pi$ to $R \pi$.

energies between hollow and filled tubes is largest in vacuum for large diameters. Increasing the screening of the surrounding leads to less strongly bound excitons, since the Coulomb interaction is more and more inhibited and the two cases of hollow and filled tubes are becoming increasingly similar to each other and obviously coincide at $\epsilon_{E} / \epsilon_{S}=1$. We also note that the exciton binding energy is very sensitive to the environment (and core) in the low-dielectric-constant range. The exciton binding energy with respect to the $2 \mathrm{D}$ case is halved, increasing $\epsilon_{E}$ from $0.1 \epsilon_{S}$ to $\sim 0.2 \epsilon_{S}$, which suggests that small changes of the dielectric environment might be revealed by optical means in this type of system, which is due to the proximity of the electronic system to the environment. We stress that excitons in InAs tubes are always less bound than excitons in GaAs or InP tubes for any considered value of the ratio $\epsilon_{E} / \epsilon_{S}$.

In Fig. 4, we also show in color scale and point size the oscillator strength of Eq. (8) as a function of diameter and dielectric configuration. For each material, the oscillator strength is normalized relative to the maximum value for the same material, so that the atomic part, $S_{0}$, of the oscillator strength cancels out. The results shown in Fig. 4 can be summarized as follows. (i) For GaAs and InP QTs, the recombination probability is larger the smaller is the diameter, while for InAs QTs, it is nearly insensitive to it. (ii) For GaAs and InP QTs, the relative oscillator strength is very weakly dependent on $\epsilon_{E}$, while for InAs QTs, it decreases for increasing ratio $\epsilon_{E} / \epsilon_{S}$. The peculiar behavior of InAs with respect to $\mathrm{GaAs}$ and $\mathrm{InP}$ in both respects must be traced to interplay between the small band gap of InAs and the very large exciton binding energy in this class of systems, making the denominator in Eq. (8) strongly dependent on $E_{b}^{x}$.

In order to further investigate the effect of the Coulomb interaction between the carriers, we plot the squared modulus of the ground-state excitonic wave function for three different dielectric environments: for a InAs tubes of diameters $D=20 \mathrm{~nm}$ (Fig. 5) and $D=100 \mathrm{~nm}$ (Fig. 6), for both hollow (top) and filled (bottom) tubes. These are two relevant cases, since the former exhibits a geometrical confinement caused by the small circumference, whereas the latter falls fully in the $2 \mathrm{D}$ regime without any confinement.

As shown in Fig. 5, the wave function for small tubes is distributed overall the circumference, best visible in the homogeneous case $\left(\epsilon_{E}=\epsilon_{S}\right)$. Reducing the screening by diminishing $\epsilon_{E}$ affects the wave functions only weakly, leading to a slightly increased localization, both of the hollow (upper panels) as well as of the filled cases (lower panels). On the other hand, both dielectric configurations lead to similar wave functions, reflected in the energies reported in Fig. 4, too.

For large tubes of Fig. 6, the wave function is no more distributed overall the circumference, but well localized. Therefore, the curvature of the tube has no effects on the exciton for larger diameters, making it fully 2D. Again, changing the dielectric configuration, by diminishing $\epsilon_{E}$ as well as by going from hollow (top panels) to filled (bottom panels) tubes, is changing the wave functions only marginally, while the respective energies are very sensitive to it (see Fig. 4). Therefore, while the diameter has a definite influence on the dimensionality of the excitonic states, changing the dielectric configuration amounts to modulating the mean screening with nearly no effects on the wave function, shifting only the energy.

\section{CONCLUSIONS}

We have studied theoretically the excitonic properties of semiconductor QTs, focusing on the influence of their dielectric environment and its interplay with structural parameters. We find that, due to the strong increase of the electron-hole interaction and ensuing very large excitonic binding which is possible in these structures, the spectral properties of excitonic absorption are strongly dependent on geometrical parameters and dielectric environment, with energies well be- 

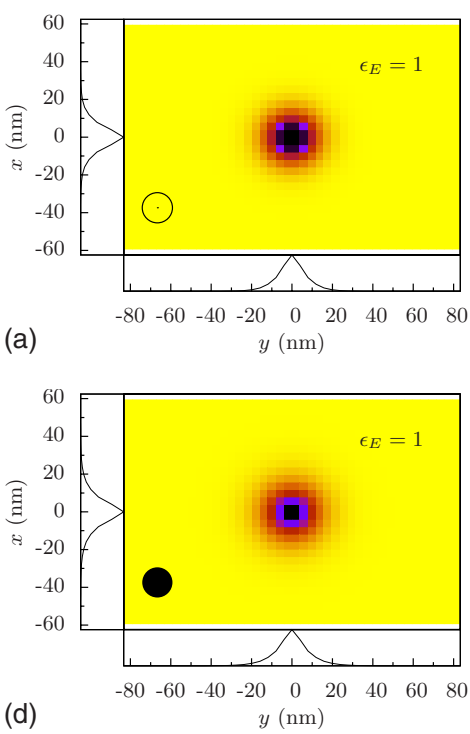
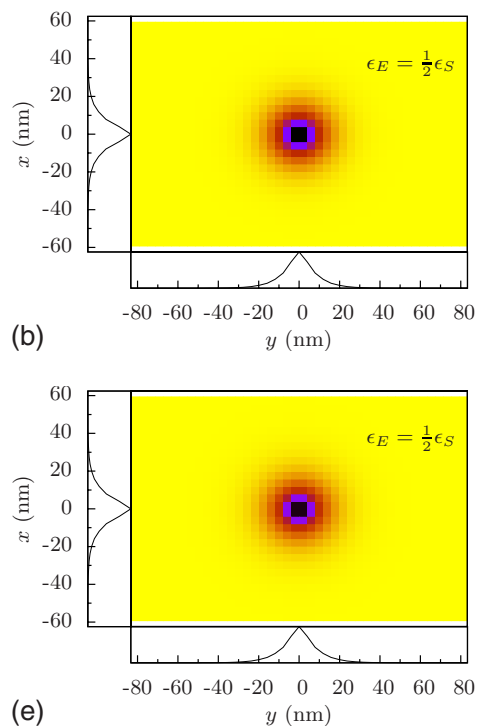
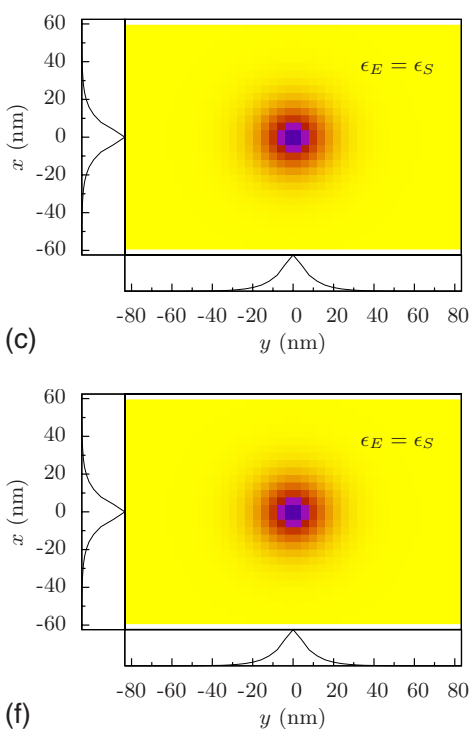

FIG. 6. (Color online) As in Fig. 5 for tubes of diameter $D=100 \mathrm{~nm}$.

low the energies of the dielectrically homogenous case which is always in the $2 \mathrm{D}$ regime for typical parameters. Calculations have been performed for InAs, GaAs, and InP. The low gap material InAs shows a peculiar behavior, since in the investigated systems, the exciton binding energy is a substantial fraction of the gap. The very large binding energies, their tunability in a wide range, and the large sensitivity of the excitonic response to the dielectric medium point to perspective applications of these systems.

\section{ACKNOWLEDGMENTS}

We thank financial support from the Italian Minister for University and Research through Grant No. FIRB RBIN04EY74 and CINECA Iniziativa Calcolo Parallelo 2009.

\section{APPENDIX: DERIVATION OF THE COULOMB INTERACTION IN A TUBE}

The inner radius $a=R-\delta$ and the outer radius $b=R+\delta$ divide the space into three regions: core $(\rho<a)$, shell $(a$ $<\rho<b)$, and environment $(\rho>b)$ with dielectric constants $\epsilon_{C}, \epsilon_{S}$, and $\epsilon_{E}$, respectively (see Fig. 1). The electrostatic potential at point $\boldsymbol{r}$ induced by an electron localized in the shell, i.e., with $a \leq \rho^{\prime} \leq b$, screened by $\epsilon_{S}$ has to obey the Poisson equation in cylindrical coordinates (with charge $e$ =1)

$$
\nabla_{\boldsymbol{r}}^{2} V_{\alpha}\left(\boldsymbol{r}, \boldsymbol{r}^{\prime}\right)=-\frac{4 \pi}{\epsilon_{S} \rho} \delta\left(\rho-\rho^{\prime}\right) \delta\left(\phi-\phi^{\prime}\right) \delta\left(z-z^{\prime}\right)
$$

Here, $\alpha$ indicates one of the three possible regions of the test charge: core $(C)$, shell $(S)$, or environment $(E)$. Eq. (A1) is solved by the ansatz

$$
\begin{aligned}
& V_{\alpha}\left(\boldsymbol{r}, \boldsymbol{r}^{\prime}\right)=\frac{1}{2 \pi^{2}} \sum_{m=-\infty}^{\infty} e^{l m\left(\phi-\phi^{\prime}\right)} \\
& \times \int_{0}^{\infty} d k \cos \left[k\left(z-z^{\prime}\right)\right] g_{m, \alpha}\left(k, \rho, \rho^{\prime}\right),
\end{aligned}
$$

where $g_{m, \alpha}\left(k, \rho, \rho^{\prime}\right)$ is the solution of the radial Poisson equation in each region $\alpha$,

$$
\frac{1}{\rho} \frac{\partial}{\partial \rho}\left(\rho \frac{\partial g_{m, \alpha}}{\partial \rho}\right)-\left(k^{2}+\frac{m^{2}}{\rho^{2}}\right) g_{m, \alpha}=-\frac{4 \pi}{\epsilon_{S} \rho} \delta\left(\rho-\rho^{\prime}\right),
$$

and can be written as a linear combination of the solutions of the homogeneous Laplace equation, i.e., modified Bessel functions of the first kind, $I_{m}(q \rho)$, and the second kind, $K_{m}(q \rho)$, with the following properties: ${ }^{42}$

$$
\begin{array}{ll}
\lim _{x \rightarrow 0} I_{m}(x)=0, & \lim _{x \rightarrow \infty} I_{m}(x)=\infty, \\
\lim _{x \rightarrow 0} K_{m}(x)=\infty, & \lim _{x \rightarrow \infty} K_{m}(x)=0 .
\end{array}
$$

Imposing that $\lim _{\rho \rightarrow \infty} g_{m, E}(k \rho)=0$ and the finiteness of $\lim _{\rho \rightarrow 0} g_{m, C}(k \rho)$, we have

$$
\begin{gathered}
g_{m, C}(\rho)=A_{m} I_{m}(k \rho), \\
\gamma_{m, S}^{\langle/\rangle}\left(\rho^{\langle/\rangle}\right)=B_{m}^{\langle/\rangle} I_{m}\left(k \rho^{\langle/\rangle}\right)+C_{m}^{\langle/\rangle} K_{m}\left(k \rho^{\langle/\rangle}\right), \\
g_{m, E}(\rho)=D_{m} K_{m}(k \rho),
\end{gathered}
$$

where $\gamma_{m, S}^{\langle/\rangle}$are no Green's functions, but solutions of the (homogeneous) Laplace equation, from which we construct the solution $g_{m, S}\left(\rho, \rho^{\prime}\right)$ of Eq. (A3) in the following. We define $\rho^{<}=\min \left[\rho, \rho^{\prime}\right]$ and $\rho^{>}=\max \left[\rho, \rho^{\prime}\right]$. Matching components of fields $E_{\|}$and $D_{\perp}$ at the interfaces is equivalent to ${ }^{43}$ 


$$
\begin{aligned}
& g_{m, C}=\gamma_{m, S}^{<}, \quad \epsilon_{C} \frac{\partial g_{m, C}}{\partial \rho}=\epsilon_{S} \frac{\partial \gamma_{m, S}^{<}}{\partial \rho} \quad \text { at } \quad \rho=a, \\
& g_{m, E}=\gamma_{m, S}^{>}, \quad \epsilon_{E} \frac{\partial g_{m, E}}{\partial \rho}=\epsilon_{S} \frac{\partial \gamma_{m, S}^{>}}{\partial \rho} \quad \text { at } \quad \rho=b .
\end{aligned}
$$

To determine the last two unknowns, we use the symmetry of the Green's function $g_{m, S}\left(\rho, \rho^{\prime}\right)$ with respect to the exchange of $\rho$ and $\rho^{\prime}$ making ${ }^{44}$

$$
g_{m, S}\left(\rho, \rho^{\prime}\right)=\gamma_{m, S}^{<} \gamma_{m, S}^{>}
$$

and normalization

$$
\gamma_{m, S}^{<}(\rho) \frac{d \gamma_{m, S}^{>}(\rho)}{d \rho}-\gamma_{m, S}^{>}(\rho) \frac{d \gamma_{m, S}^{<}(\rho)}{d \rho}=-\frac{4 \pi}{\epsilon_{S} \rho} .
$$

Defining $\alpha=\epsilon_{S} / \epsilon_{C}$ and $\beta=\epsilon_{E} / \epsilon_{S}$ and the quantities

$$
\begin{gathered}
S_{m}^{l}(k \rho)=\left.\frac{K_{m}(k \rho) I_{m}(k \rho)^{\prime}}{I_{m}(k \rho) K_{m}(k \rho)^{\prime}}\right|_{\rho=l}, \\
T_{m}^{l}(k \rho)=\left.\frac{K_{m}(k \rho)}{I_{m}(k \rho)}\right|_{\rho=l}, \\
U_{m}=T_{m}^{a}\left(S_{m}^{a}-\alpha\right)\left(S_{m}^{b}-\beta\right)-S_{m}^{a} T_{m}^{b}(\alpha-1)(\beta-1),
\end{gathered}
$$

where $I(x)^{\prime}=d I(x) / d x$ and $l=a, b$ indicates the inner and outer radii of the cylindrical shell, the coefficients in Eq. (A5) are given by

$$
\begin{gathered}
A_{m}=\frac{4 \pi}{\epsilon_{S}} \alpha T_{m}^{a}\left(S_{m}^{a}-1\right)\left(S_{m}^{b}-1\right) / U_{m}, \\
B_{m}^{<}=\frac{4 \pi}{\epsilon_{S}} T_{m}^{a}\left(S_{m}^{b}-1\right)\left(S_{m}^{a}-\alpha\right) / U_{m}, \\
B_{m}^{>}=T_{m}^{b} \frac{\beta-1}{S_{m}^{b}-1}, \\
C_{m}^{<}=\frac{4 \pi}{\epsilon_{S}} S_{m}^{a}\left(S_{m}^{b}-1\right)(\alpha-1) / U_{m},
\end{gathered}
$$

$$
\begin{gathered}
C_{m}^{>}=\frac{S_{m}^{b}-\beta}{S_{m}^{b}-1}, \\
D_{m}=1 .
\end{gathered}
$$

In particular, for two charges localized at the same distance $\rho=\rho^{\prime}=R$ from the center,

$$
\begin{gathered}
g_{m, S}(k, R, R)=\left[B_{m}^{<} I_{m}(k R)+C_{m}^{<} K_{m}(k R)\right]\left[B_{m}^{>} I_{m}(k R)\right. \\
\left.+C_{m}^{>} K_{m}(k R)\right]=\frac{4 \pi}{\epsilon_{S}}\left[\widetilde{B}_{m}^{<}+\widetilde{C}_{m}^{<}\right] \\
\times\left[\widetilde{B}_{m}^{>}+C_{m}^{>}\right] I_{m}(k R) K_{m}(k R),
\end{gathered}
$$

where we defined for clarity

$$
\begin{gathered}
\tilde{B}_{m}^{<}=\frac{\epsilon_{S}}{4 \pi} B_{m}^{<}, \\
\widetilde{C}_{m}^{<}=\frac{\epsilon_{S}}{4 \pi} \frac{K_{m}(k R)}{I_{m}(k R)} C_{m}^{<}, \\
\widetilde{B}_{m}^{>}=\frac{I_{m}(k R)}{K_{m}(k R)} B_{m}^{>} .
\end{gathered}
$$

Hence, taking Eq. (A2) in the special case $\rho=\rho^{\prime}=R$ gives the Coulomb potential for two particles localized in the shell on a cylindrical surface of radius $R$,

$$
\begin{aligned}
V\left(\boldsymbol{r}, \boldsymbol{r}^{\prime}\right) & =\frac{2}{\pi \epsilon_{S}} \sum_{m=-\infty}^{\infty} e^{\imath m\left(\phi-\phi^{\prime}\right)} \int_{0}^{\infty}\left[\widetilde{B}_{m}^{<}+\widetilde{C}_{m}^{<}\right] \\
& \times\left[\widetilde{B}_{m}^{>}+C_{m}^{>}\right] I_{m}(k R) K_{m}(k R) \cos \left[k\left(z-z^{\prime}\right)\right] d k .
\end{aligned}
$$

For $\epsilon_{C}=\epsilon_{S}=\epsilon_{E}$, this reduces to the usual form $1 / \epsilon_{S}\left|r-r^{\prime}\right|$ in cylindrical coordinates, ${ }^{44}$ while for $\epsilon_{C}=\epsilon_{S} \neq \epsilon_{E}$, Eq. (A13) reproduces the result of Ref. 45. Note that $V$ is scalable. Since all arguments in Eq. (A13) are products of lengths and momenta and thus dimensionless, only the measure $d k$ of the integral is reciprocal in length. The latter one scales with the effective Bohr length $a_{B}^{*}=\left(\epsilon_{S} / \mu\right) 0.053 \mathrm{~nm}$ and therefore $V$ itself with the effective Hartree $\mathrm{Ha}^{*}=\left(\mu / \epsilon_{S}^{2}\right) 27.21 \mathrm{eV}$.

\footnotetext{
*david.kammerlander@unimore.it

${ }^{1}$ L. J. Lauhon, M. S. Gudiksen, D. Wang, and C. M. Lieber, Nature (London) 420, 57 (2002).

${ }^{2}$ P. Mohan, J. Motohisa, and T. Fukui, Appl. Phys. Lett. 88, 133105 (2006).

${ }^{3}$ A. Fontcuberta i Morral, D. Spirkoska, J. Arbiol, M. Heigoldt, J. R. Morante, and G. Abstreiter, Small 4, 899 (2008).

${ }^{4}$ V. Y. Prinz, V. A. Seleznev, A. K. Gutakovsky, A. V. Chehovskiy, V. V. Preobrazhenskii, M. A. Putyato, and T. A. Gavrilova, Physica E (Amsterdam) 6, 828 (2000).

${ }^{5}$ O. G. Schmidt and K. Eberl, Nature (London) 410, 168 (2001).
}

${ }^{6}$ J. Noborisaka, J. Motohisa, S. Hara, and T. Fukui, Appl. Phys. Lett. 87, 093109 (2005).

${ }^{7}$ P. Mohan, J. Motohisa, and T. Fukui, Appl. Phys. Lett. 88, 013110 (2006).

${ }^{8}$ S. V. Golod, V. Y. Prinz, V. I. Mashanov, and A. K. Gutakovsky, Semicond. Sci. Technol. 16, 181 (2001).

${ }^{9}$ C. M. Lieber, MRS Bull. 28, 486 (2003).

${ }^{10}$ B. Pal, K. Goto, M. Ikezawa, Y. Masumoto, P. Mohan, J. Motohisa, and T. Fukui, Appl. Phys. Lett. 93, 073105 (2008).

${ }^{11}$ K. Goto, M. Ikezawa, S. Tomimoto, B. Pal, Y. Masumoto, P. Mohan, J. Motohisa, and T. Fukui, Jpn. J. Appl. Phys. 48, 
04C203 (2009).

${ }^{12}$ F. Jabeen, S. Rubini, V. Grillo, L. Felisari, and F. Martelli, Appl. Phys. Lett. 93, 083117 (2008).

${ }^{13}$ Y. Nagamune, Y. Arakawa, S. Tsukamoto, M. Nishioka, S. Sasaki, and N. Miura, Phys. Rev. Lett. 69, 2963 (1992).

${ }^{14}$ W. Wegscheider, L. N. Pfeiffer, M. M. Dignam, A. Pinczuk, K. W. West, S. L. McCall, and R. Hull, Phys. Rev. Lett. 71, 4071 (1993).

${ }^{15}$ T. Someya, H. Akiyama, and H. Sakaki, Phys. Rev. Lett. 74, 3664 (1995).

${ }^{16}$ F. Rossi and E. Molinari, Phys. Rev. Lett. 76, 3642 (1996).

${ }^{17}$ F. Rossi, G. Goldoni, and E. Molinari, Phys. Rev. Lett. 78, 3527 (1997).

${ }^{18}$ D. Katz, T. Wizansky, O. Millo, E. Rothenberg, T. Mokari, and U. Banin, Phys. Rev. Lett. 89, 086801 (2002).

${ }^{19}$ X. Duan, Y. Huang, R. Agarwal, and C. M. Lieber, Nature (London) 421, 241 (2003).

${ }^{20}$ A. F. Slachmuylders, B. Partoens, W. Magnus, and F. M. Peeters, Phys. Rev. B 74, 235321 (2006).

${ }^{21}$ G. Goldoni, F. Rossi, and E. Molinari, Phys. Rev. Lett. 80, 4995 (1998).

${ }^{22}$ A. Issac, C. von Borczyskowski, and F. Cichos, Phys. Rev. B 71, 161302(R) (2005).

${ }^{23}$ A. Fiore, V. Berger, E. Rosencher, P. Bravetti, and J. Nagle, Nature (London) 391, 463 (1998).

${ }^{24}$ G. Ferrari, A. Bertoni, G. Goldoni, and E. Molinari, Phys. Rev. B 78, 115326 (2008).

${ }^{25}$ G. Ferrari, G. Goldoni, A. Bertoni, and G. Cuoghi, Nano Lett. 9, 1631 (2009)

${ }^{26}$ S. Nomura, K. Tsumura, P. Mohan, J. Motohisa, and T. Fukui, Physica E (Amsterdam) 40, 1081 (2008).

${ }^{27}$ S. Nomura and K. Tsumura, Surf. Sci. 601, 441 (2007).

${ }^{28}$ This neglects the possible prismatic shape realized in some samples; see Ref. 25, and references therein.

${ }^{29}$ L. J. Sham and T. M. Rice, Phys. Rev. 144, 708 (1966).
${ }^{30}$ L. C. Andreani, Confined Electrons and Photons. New Physics and Applications (Plenum Press, New York, 1995).

${ }^{31}$ G. Bastard, Wave Mechanics Applied to Semiconductor Heterostructures (Monographies de Physique, Les Ulis, France, 1988).

${ }^{32}$ C. Deneke and O. G. Schmidt, Appl. Phys. Lett. 85, 2914 (2004).

${ }^{33}$ H. Haug and S. W. Koch, Quantum Theory of the Optical and Electronic Properties of Semiconductors, 4th ed. (World Scientific Publishing, Singapore, 2004).

${ }^{34}$ G. Bastard, E. E. Mendez, L. L. Chang, and L. Esaki, Phys. Rev. B 26, 1974 (1982).

${ }^{35}$ Although for a finite confinement the 2D binding energy would be somewhat smaller, due to exciton spill-out in the barrier material, we expect that the relative difference between the QT and its $2 \mathrm{D}$ limit is less sensitive to the details of the confinement since it is mainly due to dielectric effects.

${ }^{36}$ Small deviations from the 2D limit for GaAs and InP tubes at large diameters in Fig. 3 are a numerical artifact due to less convergence in this extreme $2 \mathrm{D}$ limit.

${ }^{37}$ T. G. Pedersen, Phys. Rev. B 67, 073401 (2003).

${ }^{38}$ D. Kammerlander, D. Prezzi, G. Goldoni, E. Molinari, and U. Hohenester, Phys. Rev. Lett. 99, 126806 (2007).

${ }^{39}$ K. Kuriyama, K. Ushiyama, K. Ohbora, Y. Miyamoto, and S. Takeda, Phys. Rev. B 58, 1103 (1998).

${ }^{40}$ A. F. Slachmuylders, B. Partoens, W. Magnus, and F. M. Peeters, Phys. Rev. B 76, 075405 (2007).

${ }^{41}$ URL: http://www.ioffe.ru/SVA/NSM/

${ }^{42}$ Handbook of Mathematical Functions with Formulas, Graphs, and Mathematical Tables, edited by M. Abramowitz and I. A. Stegun (Dover, New York, 1972).

${ }^{43}$ F. J. Garcia de Abajo and A. Howie, Phys. Rev. B 65, 115418 (2002).

${ }^{44}$ J. D. Jackson, Classical Electrodynamics (Wiley, New York, 1998).

${ }^{45}$ K. Byczuk and T. Dietl, Phys. Rev. B 60, 1507 (1999). 